\title{
Differences Between Chinese and English Thinking from the Use of Nouns in Chinese and English
}

\author{
Jingqi Tang ${ }^{1}$
}

\author{
${ }^{1}$ Eller College of Management, University of Arizona, Tucson, AZ 85721, United States \\ Email: jingqitang@email.arizona.edu
}

\begin{abstract}
Nouns play an essential role in the daily use of language, primarily identifying and naming people and things. Different language users have distinct rules and habitual methods to form words, as well as to pronounce and define grammars. These fixed and conventional language rules are influenced by speakers' thinking process, meanwhile, form speakers' particular world views and thinking modes. This article is concerned with the linguistic and thinking phenomenon that different language use and different thinking ways mutually influence each other, taking Chinese and English as typical compared instances. Based on the everyday use of nouns in two languages, this article analyses and summarizes how different word formations and use of nouns reflect different thinking ways.
\end{abstract}

Keywords: Nouns, English, Chinese

\section{INTRODUCTION}

Languages, as communication tools, are the ways people used to communicate and interact. Languages connect and reflect the world whether in oral communication, in elegant literature, or in remote information transmission. People use words to describe the vast world, naming and researching everything in nature and recording the human civilization from the ancient to the present. Not only are tools for communication, but Languages are also carriers, which transcend time and space to convey long histories and profound culture of human beings. Nowadays, there are nearly six thousand languages being recorded in the world, not counting more than one thousand endangered or extinct languages that cannot be identified and protected because of insufficient resources. Based on geographical and historical reasons, different languages are spoken by different races and different regions, making the world both unified and isolated. Speakers of the same language, especially speakers in the same regions, usually share highly similar language habits and living traditions. Without learning multiple languages, speakers of different languages generally have no ability to comprehensively understand each other's expressions because what they hear and see around their living environment is totally different. Also, different nations commonly have diverse world views and various thinking modes. These phenomena's emergence has been the foundation of many linguists' study on the relationship between using language and how we view the world, how we think, and how we feel.

\section{RELATIONSHIP BETWEEN LANGUAGES AND THOUGHTS}

Some linguists assume that languages play as the determinants of the way of thinking, but the logic of languages is absolutely not the only measure of the mode of thinking. Semantic classification and grammatical rules build meaningful languages as well as influence the formation and development of thinking. In a vivid and direct way, voice indeed constitutes language expression to give human abilities to logically convey their feelings and thoughts. However, people who are unable to use language also have thinking ability and use other ways to express their feelings and thoughts. The main opinion of the famous linguist and philosopher Wilhelm Humboldt is that thought influences and determines language, especially language structures, while language is the formative organ of thought, which means that language influences thinking modes and thinking processes [1]. Different languages have different ways to understand the world. Human thinking modes and language use reflect a great relationship of mutual influence. 
As a highly intelligent species, humans distinguish themselves from other animals by using language to express their thoughts and feelings. Humboldt defines thought is made up of some small independent units and is expressed with the use of perceptual activities. In this process, language is the symbol that humans seek to integrate those small units to create thoughts [2]. Since we are babbling infants, we have started the acquisition of our mother language and the formation of thinking by being in contact with the outside world which is mostly our family. While our bodies and brains are growing, we listen to others and receive verbal information to learn what the world is. Also, our norms and behaviors keep being influenced by what we see and hear, including language expression habits, living habits, and eventually thinking habits and world views. In the process of growth, children learn to speak, learn to classify different objects, and learn to analyze and judge, which is the establishment of the thinking process. Therefore, languages are acquired during the process of thought formation, and thinking ways are formed by means of languages.

From the name of an object to the expression of emotion and thought, the semantic meaning of a language affects a person's thinking process to understand, analyze, and judge. Words, usually the smallest meaningful unit of each language, are also the smallest independent unit of each language that can be integrated into thought. Words are used to not only name concrete objects but also describe abstract concepts. Is the naming arbitrary? Yes, we cannot find specific fixed rules that people used to name and express, especially abstract concepts like actions and emotions. However, based on our brains' memorizing and thinking processes, objects' names and their functions, behaviors descriptions and the corresponding scenes are integrated into one. We can understand what people say and can react in proper ways.

Similarly, people's intuition and habitual thoughts that are established by the semantic meaning of a language can affect people's analysis and judgment. For instance, the biological answer to what species whales belong to is that whales belong to mammals. However, whales are called "jingyu (鲸鱼)", and “yu (鱼)" means fish in Chinese. This semantic meaning gives Chinese people who do not know this scientific knowledge an intuition that whales belong to fish species. When it comes to semantic classification, like the separated departments in Walmart, there are some classification criteria but no clear boundary. This is related to a philosophical idea called Family resemblance, which is created by Ludwig Wittgenstein. This idea argues that things that share a basic common similarity may also share other overlapping similarities, but no one characteristic belongs to all things [3]. One common semantic meaning cannot describe all members in that semantic family, but people's analysis and judgment processes could be subjectively and intuitively affected because of their language use.

Different languages often carry different cultural concepts and social backgrounds, and at the same time, subtly influence different ways of thinking. Even for expressions with similar or even the same concepts and meanings, there are differences between languages. For example, first of all, unquestionably, people who only speak English and people who only speak Chinese cannot understand each other. Besides, a Chinese person who speaks English as a second or foreign language may not understand or misunderstand the expression of an English native speaker, even if this Chinese person knows every single word in the conversation. Based on cultural and thinking perspectives, there are so many possible reasons behind this situation. The expression may be a brand-new Internet slang, an idiom, or a dialect expression. Or, this Chinese person uses his/her established language analysis abilities and relevant thinking ways to understand the English expression which should be understood in English analysis and thinking modes. The latter reason is an extremely common stumbling block in the process of learning a second or foreign language because different language expressions correspond to different thinking ways. A typical instance is that English speakers and Chinese speakers have different concepts of time. Although English and Chinese speakers have horizontal concepts and use horizontal terms like "ahead", "before", "after", and "back" to talk about time, Chinese speakers commonly use vertical terms "shang (上)" and "xia (下 )" to express time as well [4]. In Chinese, "shangzhou ( 上周)” means last week, and “xiaxiageyue (下下个月)" means the month after next month. Talking about time by vertical concepts is not that common in English so that it is not so easy for English speakers to learn and understand to use "shang (上)" and "xia (下)" to describe time.

From both individual perspective and collective perspective, languages also influence the formation of world views. As the well-known British linguistic David Crystal illustrated, languages pervasively play various roles, including the way for a community's elders, leaders, and educators to transmit their stories to express their legendary experiences and social status, and the way that is used by young generations to express themselves in their social networks or on the virtual 
Internet. Hence, language stands for a guide to understanding a community's world view [5]. Through social connections and thought expressions between communities, individual thoughts are shared with others. Like Humboldt emphasizes, languages are essential to the formation of worldviews because worldviews are established when people clearly connect their own thoughts with others' and collective thinking [1]. From an individual to a society to a nation, worldviews are definitely not directly created by languages, but worldviews are embodied by languages which are a media to exhibit what the world is during people's interaction process with nature and the community.

It is undeniable that the leaped development and persistent innovation of thought are relevant to languages because human beings mainly rely on languages for viewing the world and forming thought. Semantic meaning and acquisition of languages establish people's thinking process as well as influence people's understanding, analysis, judgment, and other various thinking abilities. For the first language, acquisition of the language is a crucial step to establish thinking capacity and thinking modes, and the language expression is the manifestation of thinking ability and thinking process. Different languages have different semantic classifications and syntax rules, which form and influence speakers' thinking ways. For second and foreign languages, the learning process is generally affected by both people's mother language and the established thinking ways.

\section{COMPARISON BETWEEN CHINESE AND ENGLISH}

Chinese has the largest number of speakers in the world, while the English language is the most widely used language as an official or second language in the globe. With the development of globalization, economic activities and cultural communications become more and more frequent among different countries. As a result, mastering more than one language becomes more and more valuable. Both Chinese and English own integrated culture and long history and have experienced complex evolution. In the evolution process, these two languages have formed their own specific linguistic characteristics and will continue to unpredictably evolve with the development of human society. The differences between Chinese and English are not only reflected in linguistic concepts such as phonetics, syntax, and morphology, but also reflected in thinking processes including thinking logic and thinking modes.

\subsection{Phonetics}

In terms of phonetics, Chinese and English have their own features of syllable structure, pronunciation, and tone variation. Chinese syllables have a relatively structural order, usually consisting of an onset plus one or more coda. An onset is a consonant, and a coda is one or more vowels, or vowels plus consonants. Therefore, a syllable may have no onset, but it must have a coda. In Chinese, a Chinese character is a syllable. For example, the Chinese character "shang (上)" is made up of an onset "sh" and a coda "ang". Compared with Chinese, English has diverse syllable structures, which consist of consonants and vowels without any specific limitation of number or order. The English word "dance" is made up of multiple consonants and vowels without a fixed order. Pronunciation is also a complicated aspect to summarize and is the key and difficult points in language expression. In English, the same syllable combination can have multiple pronunciations, and rapid liaison is the essential key to read English. Pronunciation is related to tone variation as well.

Except for the syllable structure, Chinese has another unique syllable feature -- tone. In Chinese, because of the limited syllable structure, the number of valid syllables is just over 800 [6]. Many Chinese characters share the same syllables. It usually makes people confuse when listening a Chinese character without any other meaningful context. The function of tone variation in Chinese is to tell syllables that have the same structures but different meanings. According to the Rules of Hanyu Pinyin Scheme, there are four basic tones in mandarin: yinping (阴平), yangping (阳平), shangsheng (上声), qusheng (去声) [7]. Syllables “xuan (宣)" in “xuanzhi (宣纸)" (a kind of writing and painting paper used in ancient Chinese) has the same syllable structure as “xuan (选)" in “xuanze (选择)" (select). However, based on different tones and word combinations, these two characters have distinct meanings and can be easily distinguished. English also has raising and falling tones in oral English, which have different functions as Chinese. English tone is more like the expression of grammatical rules and speakers' emotions. When English speakers say, "What is that?", they are used to using a raising tone because "What is that?" is a question. If English speakers use a raising tone in a declarative sentence, they may speak with doubts, impatience, or uncertainty.

\subsection{Syntax}

Chinese and English are quite different in syntax. The main difference between Chinese and English is that Chinese has no morphological changes while English has relatively regular morphological changes to express the changes of grammatical meaning. In English, "happy" is an adjective, "happily" is an adverb, 
and "happiness" is a noun. "send" is a verb, "sends" is also a verb but is used for the third person, and "sent" is still a verb but is used for past tense. "dog" is a singular noun, and "dogs" is a noun in the plural form. Through some regular morphological changes, the word will exhibit its changes in the grammatical nature. Even though English and Chinese still have some basic similarities in grammar components such as subjectverb-object (SVO) structures in declarative sentences, they still have distinct grammar components and functions. Chinese expresses its syntax rules through an abundant auxiliary word system. In Chinese, auxiliary words “le (了)", "guo (过)" are usually used to express past tense, "zhe (着)" usually means "be doing”, and "men (们)" represents nouns plural form. Something interesting is that Chinese has three different "de (的, 地 , 得)" (sound the same but are written differently) to exhibit adjectives, adverbs, and complements. Except for this, Chinese has a rich system of quantifiers to be used with numerals to express quantitative meaning. Chinese also has a unique modal particles system to be added at the end of sentences to indicate a question, while English uses interrogative words like "what", "where", "how" in front of the sentence and inverts the order of subjects and verbs.

Compared with other aspects, syntax rules are more regular and more logical. The order of words determines the meaning of a sentence. Without a proper grammatical structure, an expression will be strange and hard to understand or express totally different meanings, or even meaningless. Word order is a fundamental grammatical rule for both English and Chinese. Although sometimes changing word order can still deliver the exact expression with the aid of context, changing word order often brings wrong messages. In Chinese, “maoyaogou (猫咬狗)” and "gouyaomao (狗 咬 猫 )” express the exact opposite information. “maoyaogou (猫咬狗)" means that a cat bites a dog, while "gouyaomao (狗咬猫)" means a cat is bitten by a dog. Chinese and English are both SVO languages, but English uses morphological changes to express different parts of speech and meanings. For example, "Anna helped them" and "Them, Anna helped" could be understood in the same way because the word "them" is in the accusative. On the other hand, like syntax rules, thinking ways and thinking processes also need logic and order. Meaningful and comprehensible language expression is built by human brain's logical thinking processes. At the same time, syntax rules can influence and solidify speakers' thinking modes and processes to make their thoughts and expression understandable.

\subsection{Morphology}

When it comes to morphology, word formation is one of the most complex linguistic phenomena and plays a significant role in affecting thinking ways. The reason is that word formation has systematic rules, just like grammar. Taking English and Chinese as an example, different languages have different main common approaches to word formation. In English, the main method of word formation is derivational morphology, which is the process of adding prefixes or suffixes to root words to create new words [8]. In general, root words are meaningful, while the affixes only change the part of speech and the meaning of the word. The adjective "happy" adding prefix "un" is "unhappy", which means "not happy". The verb "modify" adding the suffix "cation" becomes the noun "modification". Partial lexical structures are important in English word formation.

In Chinese vocabulary, a single-morpheme word (a Chinese character) can have multiple sounds and multiple meanings and is usually not enough to clearly convey all concrete meanings. Therefore, compounding is the most essential way for the Chinese to form words. Furthermore, forming Chinese vocabularies use various complex compounding rules because Chinese words have logical meanings in vocabulary structure. It seems that both derivational morphology and compounding rules basically form words by adding morpheme together. However, the difference between derivational morphology and compounding rules is that most English words are created from meaningless parts to the meaningful whole, while most Chinese words are logically created from one meaningful whole to another meaningful whole. This difference can indirectly explain that English speakers prefer analytic thinking, and Chinese speakers prefer systematic thinking. Just like syntax, word formation also has logical and ordered rules to influence thinking process and thinking expression.

\section{DAILY USE OF NOUNS AND RELEVANT THINKING WAYS}

When it comes to word formation and word use, nouns are certainly a typical class that presents the differences between English thinking and Chinese thinking. People create and use nouns not only to name concrete objects but also to define abstract concepts. English speakers and Chinese speakers usually form nouns, name things, and define concepts in distinct ways. English speakers tend to create and using nouns through extroverted, abstract, analytic, and objective thinking. However, the habitual thinking used by Chinese speakers is more conservatively, concretely, synthetically, and subjectively, and generates general Chinese characteristics of using nouns. This difference in thinking is reflected in some rules of language, as well as the use of nouns in daily life, which indicates that noun formation and noun usage show differences between English speakers and Chinese speakers on the 
basis of their different language features and thinking habits.

\subsection{Chinese Conservative Thinking versus English Extroverted Thinking}

Taking names of everyday goods as an instance, in English, a product people used to clean hair is called "shampoo". This word is originally from Hindi and means to press or smooth in Hindi. The English word shampoo does represent a liquid chemical that helps people clean their hair. However, since English mainly uses derivational morphology to form words, this noun also does not show the definition or function of this item by its literal letters. Based on the origin of this noun, it marked that English speakers borrowed this Hindi word to be the name of the item shampoo because of its original meaning of pressing and smoothing. This simple example is enough to illustrate the extroverted mode of English thinking. As a language born with borrowing words, English is an extraordinarily extroverted language. David Crystal described English as an "insatiable appetite" because English contains over $70 \%$ vocabulary which are all loanwords [9]. This deep-rooted linguistic phenomenon influences English speakers to have more extroverted thinking and more direct ways to accept foreign words and use foreign concepts. By contrast, the Chinese language obviously has fewer loanwords than English. Although Chinese still has borrowed words from several other languages such as English, Persian, and Malay, Chinese speakers have their conversion methods to translate foreign words into their own words to make borrowed words easier to be accepted and used by Chinese speakers.

\subsection{Chinese Concrete Thinking versus English Abstract Thinking}

The unique structural feature of Chinese characters typically displays the concrete and image thinking of Chinese speakers. Still using Chinese loanwords as examples, the most common methods are direct transliteration and addition of meaningful morphemes. Even though some foreign words are directly transliterated, the choices of characters are also exquisite. For example, the English word "coffee" is directly transliterated into "kafei (咖啡)". These two characters are both composed of the radical "kou (口)", which means mouth. So, this word can literally express that coffee is something for people to drink. The use of radicals is an exclusive reflection of the concrete and image thinking in Chinese. By adding meaningful morphemes, the English word "salmon" is translated into "sanwenyu (三文鱼)”, as a common everyday expression for Chinese speakers. Salmon is directly transliterated into "sanwen (三文)". In order to make it clear that "salmon" is a kind of fish, Chinese speakers add the character "yu (鱼)" because "yu (鱼)” means fish. This can interpret that Chinese speakers define borrowed words in their own introverted and conservative ways. Furthermore, adding meaningful morphemes to change words in a more acceptable way shows that Chinese speakers emphasize naming items through the clear classification of objects. Based on these characteristics, a large number of nouns used for naming indicates Chinese concrete thinking. Shampoo is named “xifalu (洗发露)" in Chinese. This name can literally tell people what the function of this item. "xi ( 洗)" has the radical “shui (水)" and means to wash, “fa ( 发)” means hair, and “lu (露)" also has the radical “yu ( 雨)" that represents “shui (水)" as well and means a kind of liquid. Therefore, it is easy for people to understand what this item is used for. Compared with Chinese, English is a relatively more abstract language because English characters themselves have no special character structures, and English words usually cannot be directly analyzed and understood through their literal and structural meanings.

\subsection{Chinese Synthetical Thinking versus English Analytic Thinking}

Chinese speakers use nouns in more synthetical ways, while the use of English nouns also reflects English analytic thinking modes. As mentioned above, adding the character "yu (鱼)" makes the loanwords "salmon" be automatically classified into fish. Chinese nouns usually use common characters to categorize objects in groups, which notably presents Chinese synthetic thinking mode. For example, Chinese uses character “ji (机)” to represent machine. “shouji (手机)” means a machine held by hands, which is a mobile phone. “zhaoxiangji (照相机)” means a machine used to take pictures, which is a camera. “paobuji (跑步机)” means a machine for running, which is a treadmill. “jisuanji (计算机)” means a machine running the algorithm, which is a computer. Using particular characters to define and categorize objects is a prominently imperative method to form words, especially nouns. This influences that Chinese speakers tend to habitually classify and compare people things during the thinking process. This also virtually provokes Chinese speakers' subjective and intuitive thinking. Meanwhile, English word formation and use of nouns usually do not have this synthetic feature. English speakers name things in a more discrete way and give different things different definitions according to their background and characteristics. English speakers usually do not habitually think about the intercommunity among mobile phones, cameras, treadmills, and computers, that they all belong to machines. 


\subsection{Chinese Subjective Thinking versus English Objective Thinking}

Being relevant to subjective thinking, Chinese speakers sometimes identify and name things more intuitively and emotionally. For example, Chinese speakers sometimes use human senses to name food, such as “xianggu (香菇)” and “chouguiyu (臭桂鱼)”. The Chinese characters “xiang (香)" and "chou (臭)" respectively mean smelling delicious and smelling stinky. These two senses feelings are very subjective and intuitive, which are rarely used by English speakers to name food. Apart from using human senses to name food, Chinese speakers also subjectively name things by personifying them or describing them. For instance, eggs laid by free range chickens are called "benjidan (笨 鸡蛋)". “ben (笨)" is generally used to describe someone who is stupid. In Chinese, flat caps or baseball caps are often called “yashemao (鸭舌帽)”. Literally, “yashemao (鸭舌帽)” means a cap looks like a duck tongue, which vividly describes the style of a cap. This kind of subjective and intuitive naming method is also uncommon in English. In English, things are mostly named according to their objective features, such as materials, components, and functions. For example, "vacuum bottle" is a water bottle taking advantage of vacuum to keep heat insulated. "cellphone" is a short name for "cellular phone", which means wireless telecommunication develops and uses to use cellular networking for connection [10]. Hence, the use of nouns to name things in English applies a more objective approach.

\section{CONCLUSION}

Acquisition and use of languages need human uses ear to hear, mouth to say, and crucially brain to think. Languages undoubtedly have effects on forming the thinking process and guiding worldviews. Different language users could eventually summarize some linguistic rules according to particular linguistic phenomena and obtain the same preferred thinking ways. So far, habitual thinking process and preferred thinking modes give people continuous abilities to develop and innovate languages. Most languages are both relevant and isolated. Because of more and more communication around the world, different languages evolve more comprehensively. Based on the specific characteristics of phonetics, syntax, and morphology, Chinese and English form their own linguistic rules and exhibit the relationship between languages and world views and thinking ways. Distinct differences between the creation and use of nouns in English and Chinese deeply demonstrate that English influences speakers to think in more extroverted, abstract, analytic, and objective ways, while Chinese speakers think and use
Chinese in a more conservative, concrete, synthetic, and subjective thinking mode.

\section{REFERENCES}

[1] Humboldt, Wilhelm, \& Humboldt, Wilhelm von. (1971). Linguistic variability \& intellectual development. (Miami linguistics series, no. 9). Coral Gables, Fla.: University of Miami Press.

[2] Humboldt, W, \& Yao, X. (2001). Wilhelm von Humboldt's Papers on Language Philosophy. Hunan Education Pubishing House.

[3] Wittgenstein, L. (1968). Philosophical investigations: The English text of the third edition. New York: Macmillan.

[4] Boroditsky, L. (2001). Does Language Shape Thought?: Mandarin and English Speakers' Conceptions of Time. Cognitive Psychology, 43(1), 1-22.

[5] Crystal, D. (2000). Language death. Cambridge, UK ; New York, NY: Cambridge University Press.

[6] Tiger Channel. (2020, May 13). Modern Chinese 22. [Video]. Youtube. https://www.youtube.com/watch?v=PQQX931 moq U\&list=PLf1yUUNidHbpCBNcfhPg4wHugdjbBri_\&index $=22$

[7] Hanyu Pinyin. (2020, November 4). In Baidu Baike. https://baike.baidu.com/item/\%E6\%B1\%89\%E8\% $\mathrm{AF} \% \mathrm{AD} \% \mathrm{E} 6 \% 8 \mathrm{~B} \% \mathrm{BC} \% \mathrm{E} 9 \% 9 \mathrm{~F} \% \mathrm{~B} 3 / 454$ ?fromtit le $=\% \mathrm{E} 6 \% 8 \mathrm{~B} \% \mathrm{BC} \% \mathrm{E} 9 \% 9 \mathrm{~F} \% \mathrm{~B} 3 \&$ fromid $=163790$ $41 \&$ fr=aladdin

[8] Tyler, Andrea, \& Nagy, William. (1989). The acquisition of English derivational morphology. Journal of Memory and Language, 28(6), 649-667.

[9] Postolachi, C. (2020). The Influence of Borrowings on the English Vocabulary [Articol].

[10] History of mobile phone. (2020, December 3). In Wikipedia. https://en.wikipedia.org/wiki/History_of_mobile_p hones\#Cellular_concepts 